\title{
APRENDIZAGEM TECNOLÓGICA E CONHECIMENTO NA PRODUÇÃO DO ETANOL 2G NO BRASIL: UM ESTUDO DE CASO
}

\author{
Liliana Cavalcante Soutinho \\ Especialista em Gestão Financeira, Controladoria e Auditoria (FGV), e Mestranda em \\ Economia Aplicada (UFAL). \\ Maceió, AL, Brasil. \\ Francisco Jose Peixoto Rosário \\ Doutor em economia da indústria e da tecnologia pelo IE/UFRJ e professor do curso de \\ mestrado em economia da FEAC/UFAL. \\ Maceió, AL, Brasil.
}

\begin{abstract}
RESUMO - O presente artigo tem como objetivo estudar as transformações ocorridas nos processos de aprendizagem e a dinâmica industrial do setor sucroenergético diante do surgimento da tecnologia de produção de etanol de segunda geração. O referencial analítico estará fundamentado na literatura de sistema setorial de inovação, oriundo da teoria evolucionária neoschumpeteriana. Apresenta-se uma revisão da bibliografia teórica sobre sistemas setoriais de inovação e as implicações dos processos subjacentes de aprendizagem para a trajetória de acumulação de capacidades tecnológicas junto com um levantamento da trajetória percorrida pelo panorama de pesquisa e desenvolvimento do setor sucroenergético brasileiro $\mathrm{O}$ aprendizado tecnológico é elemento central do estudo, e as análises serão feitas à luz desse conceito.
\end{abstract}

Palavras chaves: Setor Sucroenergético; Tecnologia; Dinâmica Industrial.

\section{TECHNOLOGICAL LEARNING AND KNOWLEDGE IN THE PRODUCTION OF 2G ETHANOL IN BRAZIL: A CASE STUDY}

\begin{abstract}
This article aims to study the transformations that occurred in the learning processes and the industrial dynamics of the sugar - energy sector in the face of the emergence of second generation ethanol technology. The analytical framework will be based on the literature on the sectorial system of innovation, derived from Neoschumpeterian evolutionary theory. We present a review of the theoretical literature on sectoral innovation systems and the implications of the underlying learning processes for the trajectory of accumulation of technological capacities together with a survey of the trajectory covered by the research and development panorama of the Brazilian sugar-energy sector Technological learning is the central element of the study, and the analyzes will be made in the light of this concept.
\end{abstract}

Keywords:

\section{INTRODUÇÃO}

O bioetanol de primeira geração é produzido com a mesma tecnologia desde 1908: é obtido por meio da fermentação do caldo e extraído da cana através da moagem e/ou do melaço. A segunda geração apresenta uma nova rota tecnológica, que é o processo conhecido como hidrólise, tecnologia que tem mais de um século de existência. No Brasil, ela teve as primeiras iniciativas de pesquisa na década de 1970, em processos de hidrólise ácida. Os esforços em pesquisa e desenvolvimento (P\&D) sobre a hidrólise geraram um aprendizado para todo o setor. Esse aprendizado acumulado sobre a produção de bioetanol abre caminho para o panorama atual de difusão do processo de hidrólise enzimática, que se apresenta como uma das rotas 
tecnológicas com maior potencial para se tornar dominante na produção de etanol para combustível em automóveis, além de possibilitar o aumento da produção e tornar viável o uso do etanol na indústria química, a denominada alcoolquímica (Silva, 2007).

Os primeiros esforços para difusão dessa tecnologia datam de 2013, com uma indústria em Crescentino, na Itália. No Brasil, a primeira planta em escala comercial de etanol de segunda geração (2G) entrou em operação em setembro de 2014, sendo a primeira do Hemisfério Sul a utilizar tal tecnologia. A opção de anexar a tecnologia de hidrólise enzimática a uma indústria de primeira geração já existente impulsiona essa nova tecnologia por permitir o compartilhamento do conhecimento e do know-how existentes no país sobre a produção do bioetanol de cana-de-açúcar e, dessa forma, integrar a segunda geração com a primeira.

O surgimento dessa inovação traz um novo conjunto de possibilidades para o desenvolvimento de novas trajetórias tecnológicas.

Segundo Dosi (2006), o paradigma tecnológico indica a direção em que as mudanças técnicas devem ser perseguidas e a determina as trajetórias tecnológicas. De acordo Bastos (2007), surgem dentro desse contexto as biorrefinarias, que poderão se transformar em uma indústria chave no século XXI, ao utilizarem os biocombustíveis de segunda geração como matéria-prima para produção de vários produtos químicos até então produzidos com base no petróleo, introduzindo um novo paradigma industrial.

Dentro dessa perspectiva, faz-se necessário estudar as transformações ocorridas na dinâmica industrial do setor sucroenergético dentro da ótica de sistema setorial de inovação, além de mapear as transformações na estrutura industrial existente (1G).

Poderia a ideia de transformação industrial proposta em Dosi 1984 explicar o advento do etanol $2 \mathrm{G}$ no Brasil e os desdobramentos futuros desse fato. Isso posto, a teoria leva a inferir que o surgimento de novas firmas, novas tecnologias e novos modelos de negócio podem estar alterando a estrutura de aprendizagem tecnológica do sistema setorial sucroenergético.

\section{REVISÃO DE LITERATURA}

O referencial analítico estará fundamentado na literatura de sistema setorial de inovação, oriundo da teoria evolucionária neoschumpeteriana. Apresenta-se uma revisão da bibliografia teórica sobre sistemas setoriais de inovação e as implicações dos processos subjacentes de aprendizagem para a trajetória de acumulação de capacidades tecnológicas. O aprendizado tecnológico é elemento central do estudo, e as análises serão feitas à luz desse conceito. Para acontecer uma inovação, é necessário o desenvolvimento, entendido como uma atividade 
cumulativa que gera aprendizado. $\mathrm{O}$ aprendizado tecnológico é definido como conhecimento adquirido pela empresa, através de seus trabalhos em pesquisa e desenvolvimento, que são um tipo de esforço tecnológico. Esse esforço implica em um processo de aprendizagem, o qual gera novos conhecimentos e pode ser tecnológico e relacional (FURTADO \& FREITAS, 2004). Analisa-se a contribuição de Dosi (1984) sobre transformação industrial para explicar o advento do etanol 2G no Brasil e quais desdobramentos futuros desse fato.

\section{PROCESSOS DE APRENDIZAGEM E ACUMULAÇÃO DE COMPETÊNCIAS TECNOLÓGICAS}

A aprendizagem é definida por Bell (1984), como os vários processos formais e informais pelos quais as habilidades e conhecimentos são adquiridos pelos indivíduos e convertidos, por meio deles, para o nível organizacional. Figueiredo (2001), complementa que a aprendizagem também deve ser entendida como um conjunto de processos que permite a empresa acumular capacidades tecnológicas ao longo do tempo.

O termo 'aprendizagem tecnológica' é, em geral, compreendido em dois sentidos. O primeiro refere-se à trajetória ou o caminho que segue a acumulação de capacidade tecnológica. Essa trajetória pode variar ao longo do tempo, ou seja, a capacidade tecnológica pode ser acumulada em velocidades e direções distintas. O segundo sentido refere-se aos vários processos pelos quais o conhecimento técnico é adquirido pelos indivíduos e convertido para o nível organizacional, isto é, conversão de conhecimento tácito individual em capacidades tecnológicas da empresa (BELL M., 1984); (FIGUEIREDO, 2001).

A noção de aprendizado utilizada neste trabalho está de acordo com Malerba (1992), que define o aprendizado como derivado de fontes de conhecimento internas e externas, o qual resulta na ampliação do estoque de conhecimentos que se acumula na firma. Nesse contexto, a firma é um acúmulo de conhecimento produtivo e tecnológico, e está sempre em busca de soluções mais vantajosas dentre diversas oportunidades possíveis. Kogut\&Zander (1992), completam a noção de empresas como um repositório de recursos, conforme determinado pelo conhecimento social embutido nas duradouras relações individuais estruturadas por princípios de organização. E, segundoNelson\&Winter (1982), o processo decisório é influenciado pelas formas de organização e processamento interno de suas atividades, as rotinas. A tomada de decisões para Teece\&Pisano, (1994) se processa em um ambiente competitivo mutante que influencia a configuração de diversidades comportamentais das firmas, gerando um ambiente marcado por assimetrias tecnológicas capazes de explicar diferentes capacidades tecnológicas entre empresas de um mesmo setor. O que complementa o trabalho de Dosi, Freeman, Nelson, 
Silverberg, Soete, (1988) que defende que, embora haja regularidades, existem componentes estocásticos, não teorizáveis. A aprendizagem é um meio pelo qual a empresa se torna capaz de acumular capacidades tecnológicas rotineiras e/ou inovadoras.

A capacidade tecnológica é definida como os recursos necessários para gerar e gerir aprimoramentos tecnológicos (BELL \& PAVITT, 1995). Tais recursos são acumulados e armazenados não apenas em sistemas físicos (máquinas, plantas de produção, softwares, bancos de dados) e nas mentes de gerentes, engenheiros e técnicos, mas também nas rotinas e procedimentos, ou seja, no sistema organizacional da empresa (BELL \& PAVITT 1995) e (FIGUEIREDO, 2001). A competência tecnológica é definida como os recursos necessários para gerar e gerenciar a mudança tecnológica, e esses recursos estão incorporados aos indivíduos e sistemas organizacionais. A mudança tecnológica é um processo contínuo que absorve ou cria o conhecimento tecnológico, determinado por fatores externos à firma e pela acumulação de habilidades e conhecimentos intrafirma (LALL, 1992).

Segundo Figueiredo (2001), a acumulação de competências tecnológicas é fator crítico para o desempenho competitivo de empresas, e a situação é ainda mais crítica para empresas atuantes em economias em industrialização.

A mudança tecnológica no nível da firma é definida como processo contínuo para absorver ou criar conhecimento tecnológico, determinado por fatores externos à firma e pela acumulação de habilidades e conhecimento intrafirma (LALL, 1992).

Segundo Bell\&Pavitt (1995), Pavitt, (1993), Lall (1992) e (Figueiredo, 2001), diferem-se os conceitos de capacidade de produção e capacitação inovadora. O primeiro conceito está relacionado às competências de rotina, que são os recursos para produzir bens e serviços em determinado nível de eficiência, usando-se uma combinação de fatores: habilidades, equipamentos, especificações de produtos e de produção, sistemas e métodos organizacionais. A capacitação tecnológica inovadora incorpora recursos adicionais e distintos para gerar e gerenciar a mudança tecnológica (BELL \& PAVITT, 1995). Destacase que a acumulação de competências tecnológicas é questão básica para alcançar a 'maturidade industrial' e, assim, tornar-se e permanecer competitiva no mercado mundial. Figueiredo, (2001) acrescenta que é por meio da aceleração da taxa de acumulação de competências tecnológicas que as empresas em industrialização conseguem aproximar-se e/ou alcançar a fronteira tecnológica em constante mudança.

Para Figueiredo (2001), a taxa ou velocidade de acumulação dessas competências pode ser medida pelo número de anos que a empresa levou para alcançar certo nível de 
competência tecnológica, ou seja, em quantos anos a empresa atuou em determinado nível até migrar para outro nível tecnológico.

É certo que os processos de aprendizagem derivam dos processos de busca das firmas por inovações, que fazem isso, justamente, com o propósito de inovar, e o aprendizado deriva também de suas atividades rotineiras de produção. Posto isso, o aprendizado é um processo que tem a capacidade de construir competências e capacitar tecnologicamente as firmas, estimulando a produção e a mudança técnica (LUNDVALL,1992). O aprender significa construir competências e habilidades novas, e não somente ter o acesso à informação. Kogut\&Zander, (1992) diferenciam informação e know-how; a primeira é definida como o conhecimento que pode ser transmitido sem perda de integridade, uma vez que as regras sintáticas necessárias para decifrar são conhecidas; e know-how é a habilidade prática acumulada ou especialização que permite fazer algo suave e eficiente. Kogut\&Zander, (1993) afirmam que a empresa é um repositório de conhecimento que consiste em ações coordenadas e em como a informação é codificada. O conhecimento que não é facilmente transmitido e replicado traz dificuldades em termos de codificabilidade e complexidade. Codificabilidade é a capacidade de a empresa estruturar o conhecimento em um conjunto de regras e relações identificáveis que podem ser facilmente comunicados. Complexidade refere-se ao número de parâmetros que definem um sistema; podem ser acomodados, mas a um custo.

A forma com que a empresa interage com o ambiente é determinante para a construção do seu conjunto particular de conhecimentos. Lundvall, Johnson, Andersen, \&Dalum (2001) complementam que a transmissão desses conhecimentos é resultado da interação entre agentes envolvidos em um contexto sociocultural e institucional específico. Kogut\&Zander (1993) completam que uma das descobertas mais persistentes nos trabalhos sobre a transferência de tecnologia é a importância da experiência prévia.

Determinantes, procedimentos e direções da mudança técnica e a transformação industrial Dosi (1982) em seu trabalho, procura explicar os determinantes, procedimentos e direções da mudança técnica e a transformação industrial, assim como os efeitos sobre o desempenho industrial e a mudança estrutural. Para isso, sugere os conceitos de paradigma tecnológico e de trajetórias tecnológicas. Giovani Dosi desenvolve o conceito de paradigma tecnológico a partir de uma analogia ao paradigma científico de Thomas Kuhn, para então entender o desenvolvimento da tecnologia. Segundo Dosi, paradigma tecnológico pode ser definido como "um 'modelo' e um 'padrão' de solução de problemas tecnológicos selecionados, baseados em princípios selecionados, derivados das ciências naturais, e em tecnologias materiais selecionadas" (Dosi, 2006). 
O paradigma surge à medida que existe um consenso a respeito dos conhecimentos científicos pertinentes para se compreender um novo problema. Assim sendo, os paradigmas tecnológicos definem as janelas de oportunidades tecnológicas para inovações e os procedimentos básicos que permitirão exploração e descobertas.

Dosi (1984) afirma que as inovações tecnológicas que acompanham os processos de instauração de novos paradigmas possuem uma dinâmica singular e um tipo especial e decisivo de inovação, e, ao analisar os determinantes da mudança técnica que se unem ao sistema socioeconômico, destaca que, quando estabelecido um caminho tecnológico promissor, inaugura-se uma fase de desdobramentos, instituição de atividades normais de solução de problemas, que vão dar forma ao novo paradigma no plano tecnológico.

Diante do exporto acima, Dosi $(1982,1984)$ define a trajetória tecnológica como o caminho de evolução permitido por um paradigma, abrangendo mudanças marginais e contínuas, ocorridas na expansão de uma tecnologia particular a partir de um ponto de descontinuidade. Assim, a trajetória, em outras palavras, pode ser definida como resultado do desenvolvimento endógeno de um paradigma tecnológico.

"Uma trajetória tecnológica" consiste numa "atividade "normal" de resolução de problemas determinada por um paradigma", e "constitui um agrupamento de possíveis direções tecnológicas, cujos limites exteriores se definem pela natureza do próprio paradigma" (Dosi 2006, págs. 44, 45 e 46).

É sabido que a mudança de paradigma não se define integralmente no jogo de interesses imediatos da produção dos capitalistas. As "trajetórias tecnológicas" surgem com capacidades transformadoras diferentes entre si. Segundo Dosi (1984, p. 19), o "mecanismo de mercado é particularmente falho na seleção ex-ante de trajetórias tecnológicas, mesmo que opere a contento na orientação posterior da evolução da trajetória selecionada". O mercado, como mecanismo de seleção, opera nos estágios mais avançados da concorrência entre trajetórias tecnológicas distintas, dizendo pouco, entretanto, no que respeita à seleção dos mecanismos de "geração de mutações" (Dosi, 2006:50). Assim, entende-se que diferentes níveis de competência tecnológica entre as empresas são resultados de características específicas à firma e dependem das mudanças nas trajetórias tecnológicas. "Um processo de seleção dos paradigmas tecnológicos" é operado pelas "forças econômicas" juntamente com os "fatores institucional e social".

Para Dosi, Orsenigo, \&Labini (2002), a noção de trajetórias tecnológicas está associada às progressivas realizações das oportunidades inovadoras subjacentes a cada paradigma, trajetórias que podem, em princípio, ser mensuradas em termos de mudanças nas características tecno-econômicas dos "artefatos" e processos de produção. 
As idéias básicas envolvidas nesta noção de trajetórias são: Primeiro, cada conjunto de conhecimento (paradigma) molda limites e taxa e direção da mudança técnica, independente de incentivos de mercado . Em segundo lugar, a mudança técnica é parcialmente impulsionada por tentativas de lidar com desequilíbrios tecnológicos que estão sendo criados. Em terceiro lugar, portanto, não deve ser capaz de ver os padrões e desvios no processo de mudanças técnicas que levam em diferentes condições de mercado (por exemplo, em diferentes preços relativos ) e do rompimento, é principalmente correlacionados mudanças radicais nas bases de conhecimento (paradigmas) Dosi et al. (2002).

Uma trajetória tecnológica vai designar o progresso dos conhecimentos tecnológicos em função das arbitragens técnicas e econômicas que são definidas pelos paradigmas. É de supor que as oportunidades de melhoramentos tecnológicos são definidas pelo paradigma, enquanto as trajetórias são em número limitado. Não existe uma infinidade de trajetórias, apenas algumas trajetórias possíveis.

Dentro do assunto principal deste trabalho, a inovação biotecnológica, é de grande valia trazer a discussão de Dosi (1993) sobre a questão da continuidade versus uma revolução biotecnológica, ponto central de seu artigo. $\mathrm{O}$ autor afirma perceber uma descontinuidade com tecnologias tradicionais e uma modificação das bases de conhecimento nas quais a tecnologia se fundamenta, e uma mudança radical nos métodos em que a inovação biotecnológica está sendo realizada, o que leva a uma tendência a mudar as dimensões fundamentais das trajetórias tecnológicas que eventualmente ocorrerão (DOSI,1993).

A mudança radical da base de conhecimento desencadeia um processo evolutivo que é lento e implica na co-participação de velhos agentes com o surgimento de novos agentes, novas empresas; um processo em que, provavelmente, há mais descontinuidade tecnológica do que descontinuidade organizacional, no qual grandes empresas, que eram dominantes em um paradigma tecnológico precedente, através de transformação, que, frequentemente, é difícil e custosa, e feita também de erros, consegue adaptar, parcialmente, a própria organização ao novo paradigma (DOSI, 1993).

\section{O PANORAMA BRASILEIRO}

\section{A EVOLUÇÃO E O AMBIENTE INSTITUCIONAL}

A agroindústria canavieira no Brasil é marcada por forte regulação estatal desde 1930 até a década de 90, intervenção que regulava a produção e os preços entre as regiões produtoras. Ao longo dos anos 1990, houve uma mudança institucional, um processo de liberalização que 
resultou em uma progressiva redução da intervenção do governo na determinação do preço do etanol, que passou a flutuar ao sabor da oferta e da demanda.

Ao final dos anos 90, a indústria se deparou com um ambiente novo e competitivo, aberto à entrada de capital estrangeiro, e é forçada a buscar estratégias nunca antes utilizadas. As firmas foram forçadas a buscar maior competitividade, produtividade e redução de custos, já que o risco passou a estar presente no negócio. Iniciam-se, então, os esforços inovativos do setor e a reestruturação do sistema setorial de inovação. Muitos trabalhos estudaram as mudanças ocorridas nessa década, dentre eles, destacamos Rosário (2008), Belik\&Vian (2002),Shikida (2002), Moraes (2000).

Os resultados dos esforços inovativos culminaram em uma trajetória positiva de aprendizagem tecnológica, fortemente baseada em inovações incrementais. Esse caminho virtuoso de inovação/difusão levou ao aumento de produtividade e à redução dos custos de produção nas etapas agrícola e industrial, e o Brasil se tornou referência internacional nas pesquisas envolvendo bioenergia. Surge, então, um novo panorama baseado na exploração da biomassa e na emergência das biorrefinarias, e, assim, um conjunto de possibilidades tecnológicas baseadas na alcoolquímica. A indústria química desenvolve o processo de hidrólise enzimática e, com isso, a produção do etanol de segunda geração é incorporada ao setor sucroenergético.

A agroindústria sucroalcooleira é uma das mais tradicionais do país, e está vivendo um momento de mudanças industriais e institucionais, que modificam toda a estrutura do setor. As firmas existentes devem lidar com mudanças tecnológicas, e é de suma importância que os principais agentes do processo de desenvolvimento tecnológico, como as universidades, os institutos de pesquisa, empresas privadas e estatais, direcionem os esforços inovadores para a trajetória existente e deem o devido incentivo ao suporte da trajetória emergente. Afirmam Olmos, Ruester, \&Liong, (2012) que os instrumentos de políticas públicas são importantes para a consolidação de uma energia alternativa, pois já existe uma infraestrutura organizada para as energias fósseis. O bioetanol a partir da cana, apesar da sua história no Brasil, ainda não é tratado como um produto energético básico, e somente a partir de 2011 o Governo Federal passou a considerar o bioetanol como um combustível, passando a ser oficialmente regulamentado pela Agência Nacional de Petróleo, Gás Natural e Biocombustíveis (ANP), o que configurou uma tentativa de avanço na regulação do etanol.

\section{OS PRINCIPAIS ESFORÇOS RELACIONADOS À TECNOLOGIA DE ETANOL DE SEGUNDA GERAÇÃO NO BRASIL}

O primeiro esforço de pesquisa para o etanol celulósico no Brasil data dos anos 1970. Em 1977, a Fundação de Tecnologia Industrial (FTI), pertencente à Universidade de São Paulo 
(USP), abordava pesquisas que utilizavam um processo de hidrólise ácida (com ácido sulfúrico concentrado) e a madeira (eucalyptuspanicutata) como matéria-prima. A FTI operou uma planta em escala piloto dos anos 1970 a 1980.

Após essa iniciativa, em 1979, surge o projeto chamado HIDROCON (Hidrólise Contínua), desenvolvido pela Companhia de Desenvolvimento Tecnológico (CODETEC) em conjunto com a Companhia Aços Villares e a Universidade Estadual de Campinas. O projeto, que utilizava primeiro a palha de arroz e depois o bagaço de cana como matéria-prima, atingiu a escala piloto e foi descontinuado, pois os rendimentos eram baixos, o que tornava a pesquisa inviável economicamente (CARDOSO, 2008).

Outra iniciativa da mesma época foi importada e desenvolvida pela COALBRA - Coque e Álcool da Madeira S/A. O processo produtivo adotado apoiava-se na hidrólise ácida, que empregava ácido sulfúrico diluído. Criada em 1979 em Uberlândia, era uma empresa de economia mista, vinculada ao Ministério da Agricultura (COALBRA, 1983). Essa experiência fracassou devido aos elevados custos da hidrólise.

Na década de 1980, a Dedini - empresa de bens de capital para o setor sucroalcooleiro decidiu investir em pesquisa para a hidrólise ácida com alguns aprimoramentos e aplicada ao bagaço da cana-de-açúcar. Foi uma parceria entre a Dedini, o Copersucar e a Fundação de Amparo à Pesquisa do Estado de São Paulo (FAPESP), em 2002, por meio do Programa Parceria para Inovação Tecnológica (PITE), que propiciou a construção de uma planta semiindustrial, instalada na Usina São Luiz, em Pirassununga (SP) (CGEE, 2009).

Percebe-se que o etanol celulósico teve uma primeira fase ligada ao Proálcool devido à criação de um mercado para o bioetanol. Porém, não houve um plano diretor para o desenvolvimento de tecnologias para esse biocombustível, muito menos via hidrólise.

Após os anos 2000, com o aumento dos preços do petróleo e de fontes energéticas fósseis, houve uma retomada do interesse pelo etanol celulósico, ocasião em que as energias alternativas voltam a ganhar espaço nas pautas de discussão e são impulsionadas pela questão ambiental, especialmente da diminuição dos gases de efeito estufa (GEE). Nessa segunda fase, a política para o bioetanol vem com uma nova configuração.

O principal estímulo é consagrado com um estudo chamado "Energias Renováveis: Etanol de Cana", que ficou conhecido como "Projeto Bioetanol", realizado em 2005. O Ministério da Ciência e Tecnologia (MCT, atual MCTI), através do Centro de Gestão de Estudos Estratégicos (CGEE), encomendou uma pesquisa ao Núcleo Interdisciplinar de Planejamento Energético (NIPE/Unicamp) a fim de demonstrar o potencial de expansão da 
produção do etanol brasileiro. A pesquisa revelou que o Brasil teria condições para substituir $10 \%$ da gasolina consumida no mundo pelo bioetanol a partir da cana-de-açúcar em 2025, e mapeou os gargalos tecnológicos a serem superados (CGEE, 2009). Essa pesquisa levou à criação do Laboratório Nacional de Ciência e Tecnologia do Bioetanol (CTBE).

Diante desse contexto, merecem destaque os seguintes esforços:

1A criação do Laboratório Nacional de Ciência e Tecnologia do Bioetanol (CTBE), vinculado ao Ministério de Ciência e Tecnologia, com objetivo de viabilizar a intenção brasileira de substituir $10 \%$ de toda a gasolina consumida no mundo até 2025 . As atividades do CTBE estão estruturadas em dois pilares: a pesquisa externa e a interna. Os estudos internos são realizados por pesquisadores do laboratório e divididos em três linhas:

a) Estudo da conversão de energia solar em biomassa, ou seja, a obtenção dos açúcares contidos na parede celular da cana;

b) Estudo da conversão de biomassa em etanol, cujo principal objetivo é obter conhecimento para a concepção de usinas integradas, que realizem a disponibilização do material lignocelulósico, hidrólise e fermentação;

c) Estudo da conversão de etanol em energia mecânica (pesquisa em motores movidos a etanol). A pesquisa externa ocorrerá via cooperação com empresas privadas, universidades e institutos de pesquisa nacionais e também internacionais.

$2 \mathrm{O}$ programa Bioenergia (Bioen), pela Fundação de Amparo à Pesquisa do Estado de São Paulo (Fapesp), com o objetivo de estimular e articular $\mathrm{P} \& \mathrm{D}$ relacionado à produção de etanol. A Bioen é composta por três empresas, seis universidades com seus diversos departamentos, quatro institutos, um centro de pesquisa, uma agência e uma associação de tecnologia, todos localizados no Estado de São Paulo. Hoje, abrange cinco linhas de pesquisa:

a) Biomassa para bioenergia, concentrada em cana-de-açúcar, incluindo genoma, bioquímica, biologia celular, fisiologia, melhoramento genético e tecnologias de cultivo de cana;

b) Processo de fabricação de biocombustíveis, cujos estudos estão voltados para o aumento da produtividade de etanol por tonelada de cana e redução do uso de água, energia e impactos ambientais do processo - é nessa linha que se encontram os estudos relacionados ao etanol celulósico;

c) Biorrefinarias e alcooquímica, focada no desenvolvimento de produtos de alto valor agregado a partir de matérias-primas renováveis (derivados químicos e petroquímica);

d) Aplicações do etanol para motores automotivos; 
e) Pesquisa sobre os impactos socioeconômicos, ambientais e uso da terra.

30 CTC iniciou o desenvolvimento de capacidades tecnológicas para produzir etanol a partir da biomassa de cana-de-açúcar (bagaço e palha) com um projeto inovador, que será integrado aos processos de geração de etanol existentes em mais de 400 usinas, atuando em parceria com a Novozymes, maior produtora mundial de enzimas (CTC, 2010).

4A criação do Plano de Apoio à Inovação Tecnológica Industrial dos Setores Sucroenergético e Sucroquímico (PAISS), com foco para o etanol celulósico. Esse programa de financiamento representa um avanço no apoio à ciência e tecnologia da produção do etanol, em seus vários aspectos. Além do mais, seu objetivo é proporcionar articulação entre governo e os atores do setor sucroalcooleiro. Essa é uma iniciativa conjunta por parte do Banco Nacional de Desenvolvimento Econômico e Social (BNDES) e da Financiadora de Estudos e Projetos (FINEP) para o fomento de projetos de novas usinas e destilarias que utilizem novas tecnologias para a produção de energia a partir da cana-de-açúcar (biomassa), na tentativa de gerar maior coordenação e integração do apoio financeiro disponível.

Como resultado dos esforços em pesquisa e desenvolvimento dos projetos relacionados à produção e comercialização da nova tecnologia, derivados da iniciativa privada, nos dias atuais, já entraram em operação as três primeiras usinas comerciais para produção de etanol de segunda geração listadas abaixo:

a.GRANBIO, em parceria com a Usina Caeté, localizada no município de São Miguel dos Campos, no Estado de Alagoas, com capacidade de produção estimada em 82 milhões de litros/ano. Firmou parcerias com a Beta Renewables, que licenciou a tecnologia Proesa para construção e operação da primeira planta de segunda geração do Brasil; com a DSM, gigante holandesa que tem acordo para fornecimento de leveduras industriais para fermentação de etanol celulósico; e com a multinacional dinamarquesa Novozymes, a fornecedora de enzimas para hidrólise de celulose.

b.Usina Boa Vista, em parceria com a PETROBRAS, localizada no município de Quirinópolis, em Goiás, com capacidade de produção de 40 milhões de litros/ano.

c.RAIZEN, com a planta industrial localizada na Usina Costa Pinto, em Piracicaba (SP). Sua operação está prevista para o final do ano - safra 2014/2015, com capacidade de 40 milhões de litros/ano. Firmou parceria com a canadense Iogen Energy, empresa com experiência no desenvolvimento de biocombustíveis celulósicos; e com a empresa dinamarquesa Novozymes, para utilização da tecnologia de enzimas específica para a produção do etanol de segunda geração nessa etapa. 


\section{PRINCIPAIS PROCESSOS DE APRENDIZAGEM OBSERVADOS}

Ao estudar e analisar os processos de produção referente a cada tecnologia e as informações relativas a esforços de aprendizagem coletadas foi possível construir um comparativo com duas trajetórias tecnológicas de setor a luz da estrutura desenvolvida Bell \&Pavitt (1995) e adaptado por figueiredo $(2001,2003)$ para exame do processos de aprendizagem do sucroenergético.

Figueiredo relaciona os principais processos de aprendizagem em 4 grupos distintos: aquisição interna de conhecimento, aquisição externa de conhecimento, codificação de conhecimento e socialização do conhecimento. Dentro desses grandes grupos será listada e avalia os esforços desenvolvidos pelas empresas do setor, surge então uma matriz comparativa.

\begin{tabular}{|c|c|c|}
\hline Aquisição externa de conhecimento & $\begin{array}{l}\text { Metal - } \\
\text { Mecânica }\end{array}$ & BioQuímica \\
\hline $\begin{array}{l}\text { Contratação com consultores externos para realização de } \\
\text { projetos }\end{array}$ & Baixa & Alta \\
\hline $\begin{array}{l}\text { Contrato com consultores externos para implantação de } \\
\text { ferramentas de gestão. }\end{array}$ & Baixa & Alta \\
\hline Contato com fabricantes nacionais & Media & Media \\
\hline Contato com fabricantes estrangeiros & Baixa & Alta \\
\hline Convênio com universidades & Media & Media \\
\hline Convenio com institutos de pesquisa & Media & Alta \\
\hline Participação em seminários e congressos & Baixa & Alta \\
\hline Treinamento externo nacional & Media & Alta \\
\hline Treinamento externo internacional & Baixa & Alta \\
\hline \multicolumn{3}{|l|}{ Aquisição interna de conhecimento } \\
\hline Treinamentos internos & Media & Alta \\
\hline Busca & Alta & Alta \\
\hline Atividades de P\&D Campo & Media & Alta \\
\hline Atividades de P\&D industrial & Baixa & Alta \\
\hline Atividades de $\mathrm{P} \& \mathrm{D}$ maquinas e equipamentos & Baixa & Alta \\
\hline \multicolumn{3}{|l|}{ Codificação de conhecimento } \\
\hline Codificações e especificações de materiais e sistema & Alta & Alta \\
\hline Banco de dados para arquivo da engenharia & Alta & Alta \\
\hline Criação de padrões de projetos & Alta & Alta \\
\hline Sistemas de controle gerenciais & Alta & Alta \\
\hline \multicolumn{3}{|l|}{ Socialização do conhecimento } \\
\hline Soluções compartilhadas de problemas & Media & Alta \\
\hline Solução compartilhada de problemas junto com fabricantes & Media & Alta \\
\hline $\begin{array}{l}\text { Desenvolvimento de especificações em conjunto com } \\
\text { fabricantes }\end{array}$ & Baixa & Alta \\
\hline $\begin{array}{l}\text { Desenvolvimento de especificações em conjunto com outras } \\
\text { áreas da empresa }\end{array}$ & Baixa & Alta \\
\hline Rotação no trabalho, trabalhos em grupo & Baixa & Media \\
\hline
\end{tabular}




\section{OS INVESTIMENTOS EM PESQUISA E DESENVOLVIMENTO DE CADA ETAPA DO PROCESSO E SEUS AGENTES.}

As parcerias e os esforços de P\&D em cada etapa do Negócio

“A GranBio é uma empresa brasileira de biotecnologia que cria soluções para transformar biomassa em produtos renováveis, como biocombustíveis e bioquímicos. Com um modelo inovador de negócios, a companhia é a única do setor que atua do começo ao fim da cadeia produtiva - da matéria prima à distribuição do produto final, integrando tecnologias próprias e de parceiros."

\section{Matéria Prima}

O inicio da cadeia produtiva é a produção de matéria prima, ou seja, a produção de Biomassa. Dado o surgimento de um novo paradigma tecnológico, a biotecnologia, a literatura o sugere como direcionador do progresso técnico, A mudança de um paradigma geralmente implica a mudança da trajetória tecnológica (DOSI, 1988).

A definição de Dosi (1982) para trajetória tecnológica é dada pelo o conjunto de atividades implementadas para a solução de um problema definido no âmbito do paradigma tecnológico. A partir das perspectivas de solução de problemas organizam-se os programas de pesquisa tecnológica. Através do advento do aperfeiçoamento dos atributos técnicos e econômicos de determinada tecnologia há o avanço da trajetória tecnológica.

Quando uma trajetória é selecionada ela apresenta impulso próprio que contribui para definir as direções em que a atividade de resolução do problema se move, sendo denominada de trajetória natural do progresso técnico (NELSON; WINTER, 2006).

A teoria permite perceber claramente os desafios do novo paradigma, dada trajetória escolhida pela Granbio, produção de Etanol de biomassa, e os caminhos que estão sendo percorridos para buscar alternativas para solução de problemas. Sendo o primeiro desafio a produção de matéria prima, BIOMASSA, percebe-se a fonte escolhida foi a CANA DE AÇÚCAR, escolha justificada principalmente por disponibilidade e produtividade dentro do Brasil, o novo paradigma modifica o foco central do desenvolvimento de pesquisa dentro dessa cultura que deixa de ser o teor de sacarose e passa a ser a produção biomassa, ou seja a celulose.

Dada a necessidade de produzir mais e melhor biomassa da cana de açúcar a Granbio desenvolve através de sua subsidiária Biovertis, na estação experimental localizada em Alagoas, pesquisa de melhoramento genético varietal em parceria com o Instituto Agronômico de Campinas (IAC) e a Rede Interuniversitária para o Desenvolvimento do Setor Sucroenergético (Ridesa), através da universidade federal de Alagoas e o programa PMGCA. 
Ambas instituições possuem uma trajetória de acumulação de competência tecnologia da tecnologia de primeira geração que pode ser aproveitada para o novo desafio imposto pela segunda geração. Segundo (BELL \& PAVITT, 1995) a competência tecnológica é definida como os recursos necessários para gerar e gerenciar a mudança tecnológica e esses recursos estão incorporados aos indivíduos e sistemas organizacionais.

Como primeiro resultado dos esforços foi desenvolvida a CanaEnergiaCanaVertix®. A variedade desenvolvida a partir do cruzamento genético de tipos ancestrais e híbridos comerciais de cana-de-açúcar, de sementes em bancos de germoplasmas do Brasil e do mundo. A canaEnergia da Granbio é mais robusta, com maior teor de fibra. Uma de suas maiores vantagens é poder ser plantada em áreas degradadas de pasto - não competindo, assim, com alimentos - e colhida em qualquer período do ano porque a região tem um microclima ideal para hibridização e seleção da variedade.

É mister destacar que há P\&D de outras fontes de biomassa, como Sorgo, Capim elefante, Milho e Eucalipto.

Para a planta industrial BIOFLEX 1 a fonte de biomassa escolhida foi a palha da cana de açúcar, que não é utilizada na indústria de primeira geração e era desperdiçada nas lavouras de cana de açúcar. Estrategicamente a planta industrial foi instalada junto a usina Caeté do Grupo Carlos Lyra, pois garante a sinergia entre a produção de $1 \mathrm{~g}$ e $2 \mathrm{~g}$ e a localização é estratégica por ser próxima a cultivares mecanizáveis que garantem a disponibilidade para o fornecimento/compra de palha de cana de usinas de 1G. Diante da solução encontrada para o fornecimento de biomassa, surge então mais um problema tecnológico que é a colheita, armazenamento e processamento de palha de cana-de-açúcar. Como solução foram feitas parcerias com as empresas CNH, Valtra e Implanor que são tracionais na produção de maquinas e implementos agrícolas para o desenvolvimento de soluções para a palha da cana.

\section{CONSIDERAÇÕES FINAIS}

É mister que a inovação é importante para a sobrevivência e renovação da estrutura dinâmica de uma economia, ressaltamos que o governo tem o papel fundamental de incentivar e estimular as inovações, a promoção de inovações é fundamental para o desenvolvimento econômico de uma região.

Como a energia desempenha um papel central para o desenvolvimento socioeconômico, os países que estiverem à frente do desenvolvimento dos biocombustíveis, terão vantagens competitivas e asseguram o desenvolvimento econômico. O Brasil vem buscando um melhor aproveitamento dessa matéria prima (cana de açúcar) para a produção de etanol, pois o fato de 
já possuir uma agroindústria destinada a esse setor a qual se articula com muitos outros setores da economia, promove o desenvolvimento em diversas áreas e faz com que o desenvolvimento local seja estimulado, aproveitando a logística, o aprendizado e todo material já existente.

Vale destacar que ainda são os primeiros desafios dessa trajetória e a cada problema há diversos caminhos e a cada solução há diversas oportunidades que leva a outros problemas tecnológicos. As soluções por hoje encontradas ainda estão em desenvolvimento e outros problemas relacionados à biomassa estão por vir.

Portanto é necessário ver que as políticas de inovação são muito mais importantes para o desenvolvimento econômico, pois além de estimular a competitividade, através do fortalecimento da capacidade de aprendizado das empresas, promove também o desenvolvimento local, já que elas podem dinamizar um setor estagnado (como é o caso do setor sucroalcooleiro de Alagoas) e que sem o incremento dessa inovação estaria fadado à falência diante do cenário nacional.

É de suma importância que os principais agentes do processo de desenvolvimento tecnológico, as universidades, os institutos de pesquisa, e o P\&D empresas privadas e estatais direcionem os esforços inovadores incrementais na trajetória existente e o devido incentivo ao suporte da trajetória emergente.

\section{REFERÊNCIA BIBLIOGRAFICA}

ADLER, M.; ZIGLIO, E. Gazing into the Oracle: The Delphi method and its application to social policy and public health. Kingsley Publishers., 1996.

ANDRÉ, M. ANDRÉ, Marli Eliza Dalmazo Afonso de. Estudo de caso em pesquisa e avaliação educacional. Brasilia: Liberlivros, 2005. p. 7-70 p.

BASTOS, V. D. Etanol, Alcooquímica e Biorrefinarias. Rio de Janeiro. 2007.

BELIK, W.; VIAN, C. E. D. F. Desregulamentação Estatal e Novas Estratégias. In: MORAES, M. A. D.; SHIKIDA, P. Agroindústria Canavieira no Brasil: evolução, desenvolvimento e desafios. São Paulo : Atlas, 2002.

BELL, M. \&. P. K. The development of technological capabilities. Technology and International Competitiveness. Washington: The World Bank, 1995.

BELL, M. Learning and the accumulation of industrial technological capacity in developing countries. King, K. \& Fransman, M. (eds.). Technological capability in the Third World, 1984.

BITTENCOURT, P. PADRÕES SETORIAIS DE APRENDIZAGEM DA INDÚSTRIA BRASILEIRA: UMA ANÁLISE EXPLORATÓRIA. Revista Brasileira de Inovação, Campinas (SP), p. p. 37-68, janeiro/junho 2012, 2012.

CARDOSO, M. V. Aplicação da radiação de feixe de elétrons como pré-tratamento do bagaço de canade-açúcar para hidrólise enzimática da celulose, 2008.

CGEE. Bioetanol combustível: uma oportunidade para o Brasil. BRASILIA. DF. 2009.

COALBRA. Questões básicas sobre o etanol de madeira. BRASILIA. 1983.

REVISTA ECONOMIA POLÍTICA DO DESENVOLVIMENTO

Maceió - AL

V.3 N.5.

DEZEMBRO/2016

P. 22-38

Página-36 
DOSI. Algunas questões sobre inovação Biotecnologica. Ensaios FEE, Porto Alegre, p. 368-371, 1993.

DOSI, G. Technological paradigms and technological trajectories: A suggested interpretations of the determinants and directions of technical change. Research Policy, p. v.11, p.147-162, 1982.

DOSI, G. Technological paradigms and technological trajectories: A suggested interpretations of the determinants and directions of technical change. Research Policy, p. v.11, p.147-162, 1982.

DOSI, G. Tenical Change and Industrial Transformation. Londes; Macmillan, 1984. Londres. 1984.

DOSI, G. Sources, Procedures, and Microeconomic Effects of Innovation.. Journal of Economic Literature, 1988. v. 26 p.1.120-1.171.

DOSI, G. Mudança técnica e Transformação Industria. campinas: unicamp, 2006.

DOSI, G. et al. The nature of the innovative process, 1988.

DOSI, G.; ORSENIGO, L.; LABINI, M. S. T. Technology and the economy. LEM working paper, 2002.

FIGUEIREDO, P. N. . Technological Learning and Competitive Performance, Cheltenham, UK, 2001.

FREEMAN, C. Japan: a New National System of Innovation?. In: DOSI, G., et al. Technical Change and Economic Theory. Londres: printer, 1988.

FREEMAN, C. The 'National System of Innovation' in historical perspective. Cambridge Journal of Economics, p. v.19. n.1, p. 5-24, 1995.

FREEMAN, C.; PEREZ, C. The diffusion of technical innovation and changes of technoeconomic paradigm. Conference on Innovation Diffusion. veneza: [s.n.]. 1986.

FREEMAN, C.; PEREZ, C. Structural crises of adjustmet: business cycles and investment behaviour. In: DOSI, G. E. A. Techincal change and economic theory. Londres: Pinter Publishers, 1988.

FURTADO, A. T.; FREITAS, A. G. Nacionalismo e Aprendizagem no programa de águas profundas da Petrobras. Revista Brasileira de Inovação, p. 55-86, 2004.

KOGUT, B.; ZANDER, U. Knowledge of the Firm, Combinative Capabilities, and the Replication of Technology., 1992.

KOGUT, B.; ZANDER, U. Knowledge of the firm and the evolutionary theory of the multinational corporation. [S.1.]: [s.n.], 1993.

LALL, S. Technological Capabilities and Industrialization.[S.1.]: World Development, 1992.

LASTRES, H. M. M.; FERRAZ, J. C. Economia da informação, do conhecimento e do aprendizado. In: LASTRES, H. M. M.; ALBAGLI, S. (. Informação e globalização na era do conhecimento. Rio de Janeiro : Campus , 1999. p. cap.1, p. 27-57.

LÜDKE, M.; ANDRÉ, M. E. D. A. Pesquisa em educação: abordagens qualitativas. São Paulo: EPU, 1986.

LUNDVALL, B. A. National Systems of Innovation: Towards a Theory of Innovation and Interactive Learning. londres: Pinter, 1992.

LUNDVALL, B. A. et al. National systems of production, innovation and competence building. DRUID Conference, Aalborg, 2001.

MALERBA, F. Learning by firms and incremental technical change. The Economic Journal, p. v. 102, n. 413, p. 845-859, jul., 1992.

MALERBA, F. Sectoral Systems of Innovation and Production.. Research Policy, p. 247-264., 2002.

MALERBA, F. Sectoral systems and innovation and technology policy. Revista Brasileira de Inovação, p. v.2, n.2, p.329-375, 2003.

MALERBA, F. Innovation and the evolution of industries. Journal of Evolutionary Economics, p. v. 16, n. 1-2, p. 3-23, Apr, 2006.

MALERBA, F.; MONTOBBIO, F. Sectoral Systems and International Technological and Trade Specialisation. DRUID SUMMER CONFERENCE. Rebild: Denmark. 2000. p. p. 15-17. 
MALERBA, F.; R., N. Catching Up: in different sectoral systems., 2008. Disponivel em: <http://dcsh.xoc.uam.mx/eii/globelicswp/wpg0801.pdf>. Acesso em: Março 2015.

MORAES, M. A. F. D. A desregulamentação do setor sucroalcooleiro do Brasil.Piracicaba: Caminho Editorial (Coleção, 2000.

NELSON, R. R. What enables rapid economic progress: What are the needed institutions? Research Policy, p. (37); 1-11., 2008.

NELSON, R.; WINTER, S. An Evolutionary Theory of Economic Change.Cambridge, MA: Harvard University Press, 1982.

NELSON, R.; WINTER, S. Uma teoria evolucionaria da mudança econômica. Unicamp, 2006. Campinas- SP: Unicamp, 2006.

OLMOS, L.; RUESTER, S.; LIONG, S. J. OLMOS, L., RUESTER, S., LIONG, S.J. On the selection of financing instruments to push the development of new technologies: Application to energy technologies. Energy Policy, 2012. vol. 43, pp. 252-266,.

PAVITT. Sectorial Patterns of Technical Change: Towards a Taxonomy and a Theory. Research Policy, p. v. 13, p. 343-373, 1984.

PAVITT, K. Technological Accumulation and Industrial Growth: Contrast Between Developed and Developing Countries. Industrial and Corporate Change, p. v.2, n.2, p.157210, 1993.7, 1993.

PAVITT, K.; ROBSON, M.; TOWSEND, J. Technological accumulation, diversification and organization in the U.K. Companies, 1945-1983. Management Science, p. p. 81-99, 1989.

ROSÁRIO, F. J. P. Competitividade e transformações estruturais na agroindústria sucroalcooleira no Brasil: uma análise sob a ótica dos sistemas setoriais de inovações. TESE, Rio de Janeiro, 2008.

SHIKIDA, P. F. A. Notas sobre Dinâmica Tecnológica e Agroindústria Canavieira no. In: MORAES, M. A. D.; SHIKIDA, P. F. A. (. Agroindústria Canavieirano Brasill: evolução, desenvolvimento e desafios. São Paulo : Atlas, 2002.

SILVA, G. “Aprendizado do etanol celulósico no Brasil: o caso do projeto Dedini Hidrólise Rápida (DHR), Campinas, 2007.

TEECE, D.; PISANO, G. The Dynamics Capabilities of Firms: an Introduction, Industrial and Corporate Change., v. V. 3, p. pp. 537-56., 1994.

TRIVIÑOS, A. N. S. Introdução à pesquisa em ciências sociais: a pesquisa qualitativa. São Paulo: Atlas, 1987.

VILLASCHI. Economia do conhecimento e do aprendizado - referência para possíveis (re) leituras da economia capixaba.. In: VILLASCHI, A. (. ). Elementos da economia capixaba e trajetórias de seu desenvolvimento. Vitória: Flor\&Cultura, 2011. p. cap. 11, p. 27.

YIN, R. K. Estudos de caso: planejamento e métodos. Porto Alegre: Bookman, 2005. 